\title{
Chronic recurrent multifocal osteomyelitis in children: a single center experience over five years
}

\author{
Erdal Sağ${ }^{1}$, Hafize Emine Sönmez ${ }^{1}$, Selcan Demir ${ }^{1}$, Yelda Bilginer ${ }^{1}$, F. Bilge Ergen ${ }^{2}$, \\ Üstün Aydıngöz $z^{2}$, Seza Özen ${ }^{1}$ \\ ${ }^{1}$ Division of Pediatric Rheumatology, Department of Pediatrics and ${ }^{2}$ Department of Radiology, Hacettepe University Faculty \\ of Medicine, Ankara, Turkey. E-mail: sezaozen@ hacettepe.edu.tr \\ Received: 21 st November 2017, Revised: 16th January 2018, 2nd May 2018, Accepted: 3rd May 2018
}

\begin{abstract}
SUMMARY: Sağ E, Sönmez HE, Demir S, Bilginer Y, Ergen FB, Aydıngöz Ü, Özen S. Chronic recurrent multifocal osteomyelitis in children: a single center experience over five years. Turk J Pediatr 2019; 61: 386-391.
\end{abstract}

Chronic recurrent multifocal osteomyelitis (CRMO) is a rare disease characterized by sterile bone inflammation. It is an orphan disease with many unclear aspects in terms of diagnosis, treatment and follow-up. The aim of this study was to report our experience of pediatric CRMO patients. Children who were diagnosed with CRMO, and were followed-up between January 2008 and January 2017, were included in this study. There were 15 CRMO patients $(8 \mathrm{M} / 7 \mathrm{~F})$ with a median age at diagnosis of 9.0 years (range: $0.6-15.0)$. Bone pain was the most common presenting symptom. All of the patients had multifocal bone lesions. Vertebrae $(66.7 \%)$ and femur $(66.7 \%)$ were the most commonly affected bones. Eight of the patients also had sacroiliitis; however, only one of them was HLA-B27 positive. Whole-body magnetic resonance imaging (MRI) was used as a diagnostic tool in 13 patients revealing bone marrow edema $(84.6 \%)$, osteitis $(69.2 \%)$, and periosteal reaction $(61.5 \%)$. All patients were initially treated with non-steroidal anti-inflammatory drugs (NSAIDs), however, disease-modifying anti-rheumatic drugs, anti-TNF agents or pamidronate were added to therapy due to inadequate treatment response. Clinical remission was achieved in 12 patients ( 1 with NSAIDs, 3 with methotrexate, 1 with pamidronate and 7 with an anti-TNF agent). During the follow-up period, relapses were observed in four patients who presented with pain and/or a newly formed bone lesion on MRI. Eventually, however, all of these patients also reached remission. CRMO is a chronic disease which may have a progressive or relapsing-remitting course. Improvement of the knowledge about this rare disease may help to enlighten the unknowns of the disease.

Key words: chronic recurrent multifocal osteomyelitis, CRMO, treatment.

Chronic recurrent multifocal osteomyelitis (CRMO) is an auto-inflammatory disease, which was first described by Giedion et al. ${ }^{1}$ and characterized by subacute or chronic sterile bone inflammation. Alternative names are chronic non-bacterial osteitis or chronic nonbacterial osteomyelitis. The main pathogenetic mechanism remains unknown; however, recent studies indicate an imbalance between pro- (IL-6 and TNF-a) and anti-inflammatory (IL-10) molecules. ${ }^{2}$ In the serum of newly diagnosed and untreated CRMO patients, the levels of IL- 6 and TNF- $\alpha$ were increased and monocytes from these patients failed to produce IL-10 under resting conditions as well as after lipopolysaccharide stimulation. ${ }^{3}$ Although a missense mutation at PSTPIP2 has been shown in murine CRMO models, neither PSTPIP2 nor any other single gene was identified in human CRMO. ${ }^{4,5}$

CRMO is an orphan disease with many unmet needs in diagnosis, treatment and follow-up. There are no validated standard criteria for the diagnosis of CRMO. Diagnosis usually depends on clinical history, physical examination and 
imaging, although in some cases, especially with a unifocal isolated lesion, biopsy is needed to rule out infections, malignancies and other possible causes. ${ }^{5}$

Although successful remission has been reported with non-steroid anti-inflammatory drugs (NSAIDs), corticosteroids and diseasemodifying anti-rheumatic drugs (DMARDs) or biological drugs, there are no randomized controlled studies for the treatment of CRMO.

The aim of the present study was to report our experience of pediatric CRMO patients regarding clinical and laboratory findings, treatment, disease activity and treatment responses.

\section{Material and Methods}

Children who were diagnosed with chronic recurrent multifocal osteomyelitis (CRMO), and were followed-up at the Pediatric Rheumatology Unit of Hacettepe University between January 2008 and January 2017, were included in this study. Clinical characteristics and laboratory findings (including radiological and pathological evaluation) were reviewed from medical charts and electronic files of the patients. In addition, treatments, treatment responses and disease activity were documented retrospectively.

All patient files were evaluated retrospectively and all patients were anonymous. When the patients were admitted to hospital, the parents gave a general consent approving anonymous data use for academic purposes. This study was approved by the Ethics Committee of Hacettepe University (September 27, 2017; GO 17/764-29).

Patients were classified as CRMO with the presence of at least one lesion consistent with osteomyelitis in the absence of infectious agents, acute phase reactants (APRs) (C-reactive protein, erythrocyte sedimentation rate), bone biopsies, and magnetic resonance imaging (MRI). ${ }^{6}$

Clinical score by Jansson et al. ${ }^{6}$ consists of seven items including blood cell count, lesion characteristics (such as symmetry or presence of marginal sclerosis), and distribution (such as vertebral, clavicular, sternal lesions or $\geq 2$ lesions proven by radiology), normal body temperature, CRP levels. ${ }^{6}$ According to this score, patients with scores from 0 to 28 are considered as most unlikely to be CRMO, scores from 29 to 38 as uncertain diagnosis, and scores of $\geq 39$ as most likely CRMO.

Due to the absence of standardized outcome measurements for CRMO, treatment response was evaluated depending on clinical and radiological findings, as well as APRs. Clinical remission was defined as the absence of subjective and objective signs of inflammation. Relapse was defined as new onset of clinical and/or radiologic findings.

\section{Statistical analysis}

SPSS software version 21 was used to perform the statistical analysis. Proportions, medians, minimum and maximum values were used where appropriate in order to present the descriptive analyses.

\section{Results}

Demographic, clinical and laboratory features of the patients (n: 15) are summarized in Table I. The median diagnostic delay was 23 (1.5-68) months. All of the patients except one suffered from bone pain, mostly affecting lower extremities $(53.4 \%)$, and six of them (40\%) had multifocal bone pain at time of diagnosis. One patient was referred to our outpatient clinic only due to limping.

All of the patients had multifocal bone lesions at the time of diagnosis. On average, each patient had six affected sites. The distribution of the affected bones has been summarized in Table II. Most commonly affected areas were femur (10 patients) and vertebrae (10 patients). Overall, $93.3 \%$ of them had lower extremity involvement, $53.3 \%$ had upper extremity involvement. In addition to bone lesions, 8 (53.4\%) patients presented with sacroiliitis. Only one patient had CRMO related extra-osseous symptom which was skin abscesses.

Ten patients had elevated ESR (normal range $0-20 \mathrm{~mm} /$ hour) and eight patients had increased CRP levels (normal range $0-0.8 \mathrm{mg}$ / 
Table I. Demographic, Clinical and Laboratory Features of Patients.

\begin{tabular}{|c|c|}
\hline Features & Results* \\
\hline Male gender & $8(53.4)$ \\
\hline Median age at onset, years* & $9.0(0.6-15.0)$ years \\
\hline Median age at diagnosis, years* & $10.5(3.0-16.5)$ years \\
\hline \multicolumn{2}{|l|}{ Presenting symptoms, n (\%) } \\
\hline Unifocal extremity or back pain & $8(53.4)$ \\
\hline Multifocal bone pain & $6(40.0)$ \\
\hline Limping & $1(6.6)$ \\
\hline \multicolumn{2}{|l|}{ Laboratory finding* } \\
\hline Hemoglobin, gr/dl & $12.0(9.9-13.4)$ \\
\hline $\mathrm{WBC}, / \mathrm{mm}^{3}$ & $7,800(6,300-20,600)$ \\
\hline Platelet, $/ \mathrm{mm}^{3}$ & $407,000(215,000-854,000)$ \\
\hline Erythrocyte sedimentation rate, $\mathrm{mm} /$ hour & $29(2-120)$ \\
\hline C-reactive protein, $\mathrm{mg} / \mathrm{dl}$ & $0.98(0.25-14.7)$ \\
\hline Bone biopsy, n (\%) & $6(40.0)$ \\
\hline Median clinical score ${ }^{*, \#}$ & $43(21-53)$ \\
\hline \multicolumn{2}{|l|}{ Whole-body MRI findings $(\mathrm{N}=13), \mathrm{n}(\%)$} \\
\hline Bone marrow edema & $11(84.6)$ \\
\hline Active osteitis & $9(69.2)$ \\
\hline Periosteal reaction & $8(61.5)$ \\
\hline Bone expansion & $2(15.3)$ \\
\hline Lytic lesion & $1(7.6)$ \\
\hline Enthesitis & $1(7.6)$ \\
\hline Sacroiliitis, n (\%) & $8(53.4)$ \\
\hline Remission, n (\%) & $12(80.0)$ \\
\hline Non-steroid anti-inflammatory drugs (NSAID), n (\%) & $1(6.6)$ \\
\hline Pamidronate & $1(6.6)$ \\
\hline Methotrexate & $3(19.8)$ \\
\hline Anti-TNF agents & $7(46.6)$ \\
\hline
\end{tabular}

*: Values are presented as median (minimum-maximum).

": Details are given in the methods (Reference 6).

dl). Patients with sacroiliitis were screened for the presence of human leukocyte antigen (HLA)-B27, but it was positive in only one patient. All except two of the patients underwent whole body (WB) MRI at diagnosis; findings are presented in Table II. Biopsy from bone lesions was performed in six (40\%) patients. All of the biopsies were performed before 2014. All of the biopsy samples revealed osteomyelitis without presence of infections and showed evidence of mixed inflammatory infiltration and sclerosis.
Median (min.-max.) clinical score described by Jansson et al. ${ }^{6}$ was 43 (21-53). Only one patient had a clinical score lower than 28, three patients had scores ranging 28 to 38 , and 11 patients had scores higher than 38 . The patient with the clinical score under 28 had a bone biopsy which was compatible with CRMO. Only one out of 3 patients, who were classified as uncertain diagnosis according to clinical score, had a biopsy-proven diagnosis. 
Table II. Distribution of Affected Bones on Magnetic Resonance Imaging.

\begin{tabular}{lc}
\hline Affected bones & Results, $\mathrm{n}(\%)$ \\
\hline Vertebra & $10(66.7)$ \\
Femur & $10(66.7)$ \\
Sacroiliitis & $8(53.4)$ \\
Pelvis & $6(40.0)$ \\
Tibia & $6(40.0)$ \\
Humerus & $5(33.3)$ \\
Sternum & $4(26.7)$ \\
Clavicle & $4(26.7)$ \\
Radius & $2(13.3)$ \\
Ulna & $2(13.3)$ \\
Mandible & $1(6.6)$ \\
Talus & $1(6.6)$ \\
Calcaneus & $1(6.6)$ \\
\hline
\end{tabular}

\section{Treatments and outcomes}

All patients were initially treated with NSAIDs. One patient achieved remission with only NSAIDs. However, DMARDs were needed to be added to the treatment in 13 patients (methotrexate in 12, sulfasalazine in one) after the failure of 3 months of NSAID therapy. The treatment was switched to anti-tumor necrosis factor (TNF) (etanercept) agent in 8 patients due to inadequate response of DMARDs after a mean duration of 4.6 months. Three patients received pamidronate. Of these three patients, two were administered pamidronate due to relapse with anti-TNF treatment, and one received pamidronate after non-response to DMARDs.

The median (min.-max.) follow-up time was 5.0 (1.0-9.5) years. Patients were evaluated every six months for clinical remission. Among 15 patients, 12 (80\%) achieved clinical remission within 6-24 months. Remission was confirmed in eight patients with radiologic findings. Although clinical remission was achieved, four patients still had active lesions on control MRI. Seven patients achieved clinical remission with anti-TNF (six with etanercept, one with infliximab; mean duration to reach remission was 11.5 months), three patients with methotrexate (mean duration to reach remission was 17.5 months), one with pamidronate (reached remission in 6 months), and one with NSAIDs (reached remission in 2 months). Three patients continued to have clinical and radiologic active disease, despite anti-TNF treatment in two patients and pamidronate in one patient. During the follow-up period, relapses were observed in four patients who presented with pain and/or a newly formed bone lesion on MRI. Eventually, however, all patients reached remission.

Collapse and fracture in vertebrae were detected in three patients at the time of diagnosis. These patients had a long diagnostic delay time ranging from 23 to 36 months.

\section{Discussion}

In the presented study we summarized the clinical characteristics, radiological findings, treatments, and outcome in pediatric CRMO patients.

Few studies have been published to describe the disease characteristics of CRMO. Recently the French group ${ }^{7}$ has reported the largest cohort of CRMO including 178 patients. According to this study, there is a female predominance (F/M: 2/1) with a mean age of symptom onset of 9.8 years and a mean age of diagnosis of 16.4 years. The diagnostic delay time was a median 17.3 months. Painful bone lesions were the most common symptom where nearly $70 \%$ of the patients had multifocal painful osseous lesions with the involvement of lower limbs (47\%), pelvis (\%16), clavicles $(10 \%)$ and vertebrae (8\%) at diagnosis. ${ }^{7,8}$ In our cohort, diagnostic delay was longer (23 months). Interestingly, although only $40 \%$ of our patients suffered from multifocal bone pain, all patients had multifocal lesions on WB-MRI at the time of diagnosis.

The distribution of the lesions was also somewhat different from the French cohort. Almost all of our patients had lower extremity involvement (93.3\%); vertebral (66.7\%) involvement was more common than in the French cohort. Three of the patients with vertebral involvement $(30 \%)$ presented with collapse and fracture. Previously, increased risk of vertebral fracture was described in the French cohort among patients with vertebral lesions. ${ }^{7}$ They suggested the use of more 
aggressive treatment in patients with vertebral lesions to prevent complication. Compatible with this result, in our cohort the patients with vertebral involvement also had a progressive course.

Extra-osseous manifestations such as inflammatory bowel disease, palmoplantar pustulosis, acne and psoriasis may also be seen during the course of CRMO.$^{5,7}$ In previous studies, it was reported that extra-osseous manifestations accompanied the bone lesions ranging from $12 \%$ to $31 \% .^{7-8}$ However, in this study, only one patient had an extra-osseous symptom in the form of skin abscesses. On the other hand, about half of the patients (53.4\%) presented with sacroiliitis. All but one of the patients were HLA-27 negative. In fact, some authors suggest that CRMO may even be considered within the spectrum of spondyloarthritides. ${ }^{9}$ However, it is generally agreed that CRMO should be classified as an autoinflammatory disease. ${ }^{10}$

Inflammatory markers such as ESR, CRP and WBC count are mildly elevated; on the other hand, if the inflammatory markers are very high, infections and malignancies should be suspected. ${ }^{5}$ In contrast, $67 \%$ of the French CRMO cohort presented with elevated acute phase reactants. ${ }^{7}$ However, the authors suggested that it might depend on selection bias as their hospitals were tertiary centers. In the presented study, $66.7 \%$ patients had elevated APRs which was compatible with the findings in the French cohort.

Plain X-rays may be normal at early phases of the disease and radionuclide bone scans have been used to identify silent bone lesions. However, WB-MRI, showing characteristic bone marrow edema, is more sensitive than radiographs and bone scans for both identifying the silent lesions and monitoring disease activity and also treatment responses ${ }^{11,12}$. We used WB-MRI for both diagnosis and followup. Similar to previous studies bone marrow edema, osteitis and periosteal reaction were the most common radiological features in our patients. It is important to note that 4 of our patients had active lesions on MRI although they were clinically in remission.

Bone biopsy is usually performed in patients with a unifocal lesion to rule out differential diagnosis. Recently, Jansson et al. ${ }^{6}$ have designed a score to determine whether a bone biopsy is necessary to confirm the CRMO diagnosis. The French cohort has confirmed that this score helps to prevent unnecessary bone biopsies. ${ }^{7}$ Most of our patients (73.4\%) were classified as CRMO according to the Jansson score. We performed bone biopsy in six $(40 \%)$ patients. Among six patients, only one patient had a clinical score lower than 28 , three patients had scores ranging 28 to 38. With the improvement of our knowledge about the disease, we prefer bone biopsy as the last choice for very complicated or unusually presented cases.

NSAIDs are usually the first-line treatment which provides clinical and radiological improvement in most patients. ${ }^{7,13,14}$ Corticosteroids and DMARDs (methotrexate, azathioprine, and sulfasalazine) are usually needed as an adjunctive therapy for patients who fail to response NSAIDs. ${ }^{5}$ Bisphosphonates (especially pamidronate) have been used with an increasing evidence of efficacy in reducing pain and restoring function ${ }^{5}$. Roderick et al. ${ }^{15}$ have evaluated 11 CRMO patients treated with pamidronate and demonstrated complete resolution of inflammatory lesions in $60 \%$ of them with pamidronate at one year. Three of our patients were treated with pamidronate, but only one of them reached the remission. Biologics are also used for the treatment of refractory patients. Hence TNF- $\alpha$ plays an important role at the pathogenesis of the disease, anti-TNF- $\alpha$ treatments, especially infliximab and etanercept, have been used in treatment-resistant patients and achieved clinical remission. ${ }^{5,16}$ We have previously reported successful remission with etanercept in five refractory CRMO cases. ${ }^{16}$ In the current study, six patients achieved remission with etanercept and one with infliximab.

The course of the disease is very variable, with relapsing and remitting symptoms and there is no consensus on monitoring the disease activity. WB-MRI may be used to assess activity at follow-up. ${ }^{5}$ Twelve of our patients received clinical remission within 6-24 months and radiologic remission was confirmed with WB-MRI in eight $(66.7 \%)$ of them, which was similar to results of other published cohorts. ${ }^{7,8}$ 
In conclusion, CRMO is a chronic disease which may have a progressive or relapsingremitting course. NSAIDs and anti-TNF drugs are the most efficacious treatment; however, pamidronate may also be used for refractory cases. Although there is no consensus on follow-up parameters, WB-MRI is a useful tool to show an active disease even when the patient is in clinical remission.

\section{REFERENCES}

1. Giedion A, Holthusen W, Masel LF, Vischer D. Subacute and chronic "symmetrical" osteomyelitis. Ann Radiol (Paris) 1972; 15: 329-342.

2. Hedrich CM, Hofmann SR, Pablik J, Morbach H, Girschick HJ. Autoinflammatory bone disorders with special focus on chronic recurrent multifocal osteomyelitis (CRMO). Pediatr Rheumatol Online J 2013; 11: 47.

3. Hofmann SR, Schwarz T, Moller JC, et al. Chronic non-bacterial osteomyelitis is associated with impaired Sp1 signaling, reduced IL10 promoter phosphorylation, and reduced myeloid IL-10 expression. Clin Immunol 2011; 141: 317-327.

4. Ferguson PJ, Bing X, Vasef MA, et al. A missense mutation in pstpip2 is associated with the murine autoinflammatory disorder chronic multifocal osteomyelitis. Bone 2006; 38: 41-47.

5. Roderick MR, Sen ES, Ramanan AV. Chronic recurrent multifocal osteomyelitis in children and adults: Current understanding and areas for development. Rheumatology (Oxford) 2018; 57: 41-48.

6. Jansson AF, Muller TH, Gliera L, et al. Clinical score for nonbacterial osteitis in children and adults. Arthritis Rheum 2009; 60: 1152-1159.

7. Wipff J, Costantino F, Lemelle I, et al. A large national cohort of French patients with chronic recurrent multifocal osteitis. Arthritis Rheum 2015; 67: 11281137.
8. Jansson A, Renner ED, Ramser J, et al. Classification of non-bacterial osteitis: Retrospective study of clinical, immunological and genetic aspects in 89 patients. Rheumatology (Oxford) 2007; 46: 154-160.

9. Dougados M, Baeten D. Spondyloarthritis. Lancet 2011; 377: 2127-2137.

10. McGonagle D, McDermott MF. A proposed classification of the immunological diseases. PLoS Med 2006; 3: e297.

11. Morbach H, Schneider P, Schwarz T, et al. Comparison of magnetic resonance imaging and 99mTechnetiumlabelled methylene diphosphonate bone scintigraphy in the initial assessment of chronic non-bacterial osteomyelitis of childhood and adolescents. Clin Exp Rheumatol 2012; 30: 578-582.

12. Fritz J, Tzaribatchev N, Claussen CD, Carrino JA, Horger MS. Chronic recurrent multifocal osteomyelitis: Comparison of whole-body MR imaging with radiography and correlation with clinical and laboratory data. Radiology 2009; 252: 842-851.

13. Abril JC, Ramirez A. Successful treatment of chronic recurrent multifocal osteomyelitis with indomethacin: A preliminary report of five cases. J Pediatr Orthop 2007; 27: 587-591.

14. Walsh P, Manners PJ, Vercoe J, Burgner D, Murray $\mathrm{KJ}$. Chronic recurrent multifocal osteomyelitis in children: Nine years' experience at a statewide tertiary paediatric rheumatology referral centre. Rheumatology (Oxford) 2015; 54: 1688-1691.

15. Roderick M, Shah R, Finn A, Ramanan AV. Efficacy of pamidronate therapy in children with chronic non-bacterial osteitis: Disease activity assessment by whole body magnetic resonance imaging. Rheumatology (Oxford) 2014; 53: 1973-1976.

16. Batu ED, Ergen FB, Gulhan B, Topaloglu R, Aydingoz $\mathrm{U}$, Ozen S. Etanercept treatment in five cases of refractory chronic recurrent multifocal osteomyelitis (CRMO). Joint Bone Spine 2015; 82: 471-473. 